\title{
Detailed Studies of Radiogalaxy Mrk1032
}

\author{
Hakopian S.A.*1 ${ }^{* 1}$ Dodonov S.N. ${ }^{\dagger 2}$, and Andreasyan R.R. ${ }^{\ddagger 1}$ \\ ${ }^{1}$ V.Ambartsumian Byurakan Astrophysical Observatory (BAO), Armenia \\ ${ }^{2}$ SpecialAstrophysical Observatory(SAO), Russia
}

\begin{abstract}
The first results of the observations of Mrk1032, conducted with the 6 -m telescope of SAO Russia, are given. It is shown, in particular, that a large complex of HII-regions, revealed by the integral-field spetroscopy, is the main source of UV radiation in the galaxy.
\end{abstract}

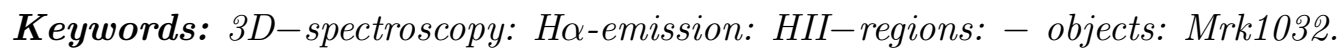

\section{Introduction}

The first publication on the object Mrk1032 (PGC008924) appeared as the manifestation of it as a "UV-excess" galaxy through the conducting of the First Byurakan Survey, FBS, (Markarian et al., 1979). The object of about $16 \mathrm{~m}(\mathrm{pg})$ apparent magnitude and 9" $\mathrm{x} 7$ " size received survey classification ds3e:. It was noted, herewith, that the west component of an interacting pare was implied, which denoted A on Fig.1 with the image from DSS. The only available optical spectrum of Mrk1032 has been obtained through the follow-up spectroscopy with 70-cm telescope, which showed emission in Balmer $\mathrm{H} \alpha$, and two forbidden lines of ionized nitrogen at $\lambda 6584$ and sulfur at $\lambda 6731$ (Denisiuk \& Lipovetskii, 1984). The very first observations of Mrk1032 conducted with RATAN radiotelescope (Sanamian \& Kandalian, 1968) revealed radioemission. Further observations (Maehara et al., 1985) and publications confirmed that the "UV-excess" Mrk1032 is a radiogalaxy too. This became the basis for attributing to the galaxy the AGN nature of presumably Sy2 type.
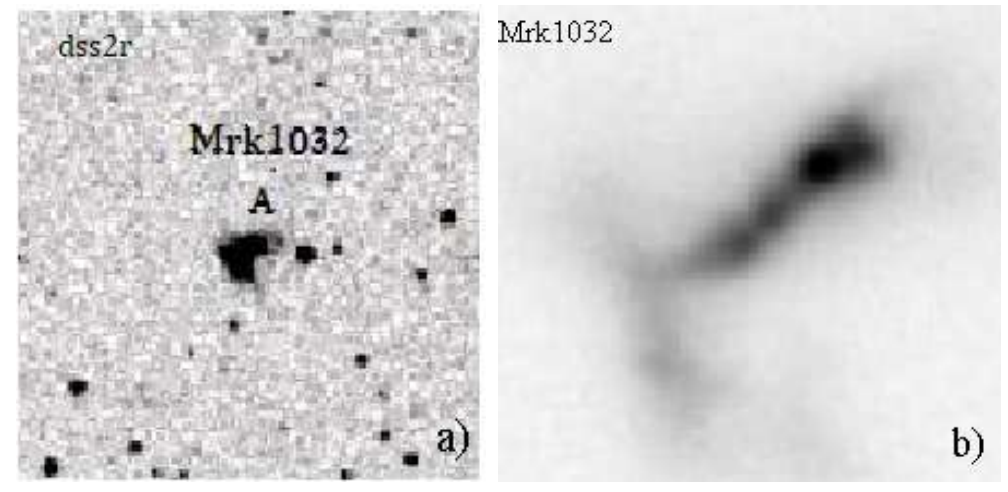

Figure 1. Image of Mrk1032: a) from DSS2r, b) from PANSTARRS red.

Due to the "high degree of UV-excess (H)" the object was included in the KISO survey catalog (Takase \& Miyauchi-Isobe, 1984) too, with morphological classification "Pair of interacting galaxy (Pi)". Cross-identifications between different surveys show sources associated with the object in GALEX, 2MASSXJ, IRAS, GAIA, NVSS and other surveys, from ultraviolet to radio. An image from the PanSTARRS survey, shown in Fig.1b, is the most detailed for now.

*susannahakopian@yahoo.com, Corresponding author

${ }^{\dagger}$ dodo@sao.ru

${ }^{\ddagger}$ randrasy@bao.sci.am 


\section{Results of integral-field spectroscopy of Mrk1032}

Aimed at detailed investigations of the galaxy we have undertaken its observations with the $6-\mathrm{m}$ telescope of SAO of Russia using the MPFSpectrograph (http://www.sao.ru/hq/lsfvo/index.html). Some results from the provided integral-field spectroscopy are presented below. Fig.2 contains: main information on the mode of observations, field of view as smoothed pixels of intensities summarized over the whole registered spectral range and the spectrum obtained in the pixel, where an intensity in Balmer $\mathrm{H} \alpha$ line, $\mathrm{I}(\mathrm{H} \alpha) \max$, has peak value. What is evident from the profiles of the most intense lines present in the spectrum, namely $\mathrm{H} \alpha$ and the forbidden doublets of nitrogen [NII]6548,6584 and sulfur [SII]6717,6731, is that the processes ongoing in Mrk1032 are not of AGN nature, but have a starburst activity. In correspondence to our scheme of activity classification (Hakopian, 2013) the object is of star-forming type galaxy in nebular phase (SfGneb).

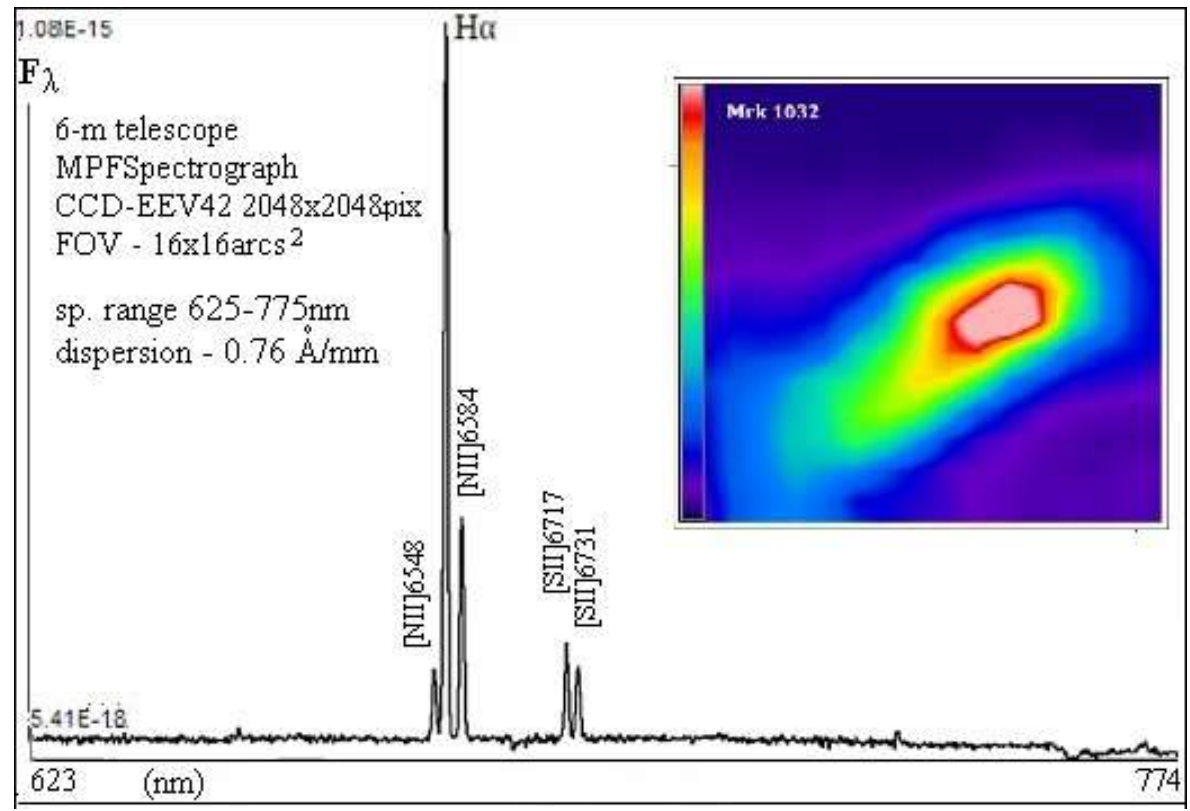

Figure 2. General data on the mode of observations, FOV and the spectrum in the pixel $\mathrm{I}(\mathrm{H} \alpha) \max$.
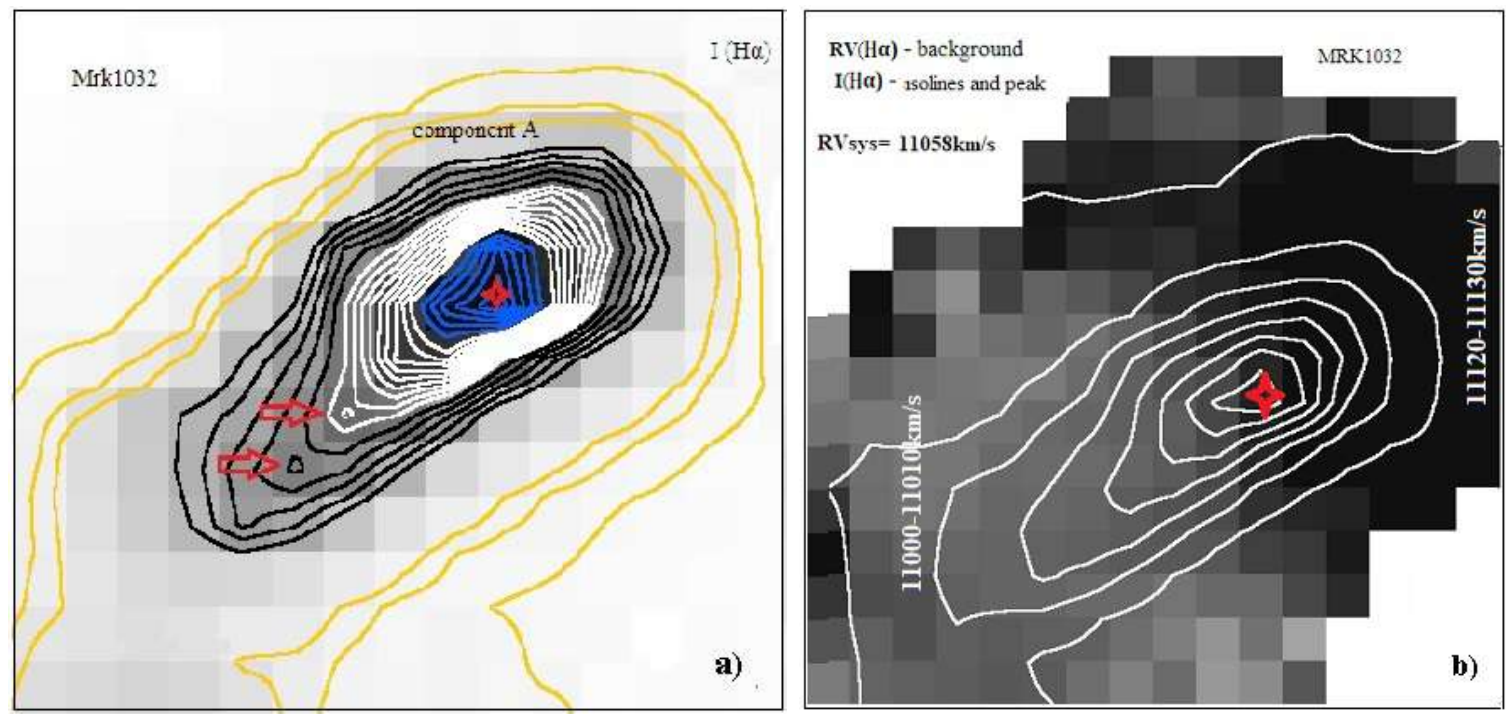

Figure 3. a) $\mathrm{H} \alpha$-intensity distribution over the entire FOV; b) $\mathrm{H} \alpha$ radial velocities across the main part of the FOV with some numerical data. 
Surface distribution of $\mathrm{H} \alpha$-emission, first of all, allowed to reveal HII-regions of a galaxy directly connected to star-formation processes. Fig.3 illustrates the observed for Mkn1032 FOV as a gray scaled background with superimposed isolines, both reflecting $\mathrm{H} \alpha-$ line intensities. Two small HII-regions with centers indicated by arrows, and a large HII-zone with the peak intensity pixel $\mathrm{I}(\mathrm{H} \alpha)$ max marked by a cross, are differentiated. On the whole their locations repeat positions of condensations, which are visually differentiated from the given on Fig.1b image. Herewith, upon removal from the peak $\mathrm{I}(\mathrm{H} \alpha)$ max the shapes and different gradients of the isolines, as seen on Fig.3a, indicate that the large HII-zone is not monosyllabic. The same conclusion is drawn from the analysis of the velocity field.

Fig.3b gives distribution of radial velocities $\mathrm{RV}(\mathrm{H} \alpha)$ as background gradations with superimposed $\mathrm{H} \alpha$ - isolines. The values of systemic velocity, obtained for the cross marked pixel of peak $\mathrm{H} \alpha$-intensity and the values averaged over two border areas are also given. A sharp transition from the smaller values of $\operatorname{RV}(\mathrm{H} \alpha)$ to the larger ones is visually seen on this map due to changing in the background gradations reflecting them. Passing through the marked peak its trajectory divides the HII-zone into two parts and evidences the presence of at least a double structure aligned along the line of sight.

\section{Conclusions}

These are the first results from our observations of Mrk1032, still incomplete, but giving answers to the two remaining open questions.

First, the object is not a galaxy of Sy2 type, which follows from the spectral line profiles. According to our classification scheme, this is a star-forming galaxy in the nebular phase, SfGneb.

Second, that the eastern tail of the object is too diffuse and has not enough dense structural features to be considered as a separate component. The distribution over the whole field of the galaxy in the continuum, having only one peak, confirms it.

Thus, the Mrk1032 is not doubled, not interacting, but a galaxy of irregular morphology. A large complex of HII-regions in its west part, originally designated A, is the main source of its UV radiation.

\section{References}

Denisiuk E. K., Lipovetskii V. A., 1984, Astrofizika, 20, 525D

Hakopian S. A., 2013, arXiv:1403.0127,

Maehara H., Inoue M., Takase B., et al. 1985, PASJ, 37, 451M

Markarian B. E., Lipovetskii V. A., Stepanian D. A., 1979, Astrofizika, 13, 397M

Sanamian V. A., Kandalian R. A., 1968, Astrofizika, 4, 129A

Takase B., Miyauchi-Isobe N., 1984, AnTok, 192, 595T 